Pirineos. Revista de Ecología de Montaña

Vol. 174

Jaca, Enero-Diciembre, 2019, e048

ISSN-1: 0373-2568

https://doi.org/10.3989/pirineos.2019.174008

\title{
SEMILLAS DE MONTAÑA: RECOLECCIÓN, USOS Y COMERCIALIZACIÓN DEL PIÑÓN DE LA ARAUCARIA (Araucaria araucana) POR COMUNIDADES MAPUCHE-PEWENCHE DEL SUR DE LOS ANDES
}

\section{Mountain seeds: gathering, uses and commercialization of seeds of the monkey puzzle tree (Araucaria araucana) by Mapuche-Pewenche communities of the southern Andes}

\author{
Josefina Cortés $^{1,2}$, Isabel Ugalde ${ }^{2}$, Julián Caviedes ${ }^{1}$, José Tomás Ibarra ${ }^{1,2,3 *}$ \\ ${ }^{1}$ Laboratorio ECOS (Ecología-Complejidad-Sociedad), Centro UC de Desarrollo Local, Educación e Interculturalidad (CEDEL), \\ Campus Villarrica, Pontificia Universidad Católica de Chile, Bernardo O'Higgins 501, Villarrica, La Araucanía, Chile. \\ ${ }^{2}$ Centro de Estudios Interculturales e Indígenas (CIIR), Facultad de Ciencias Sociales, Pontificia Universidad Católica de Chile, \\ Av. Vicuña Mackenna 4860, Macul, Santiago, Chile. \\ ${ }^{3}$ Núcleo Milenio Centro para el Impacto Socioeconómico de las Políticas Ambientales (CESIEP) \& Centro de Ecología Aplicada y Sustentabilidad (CAPES), \\ Pontificia Universidad Católica de Chile, Av. Vicuña Mackenna 4860, Macul, Santiago, Chile.
}

Identificador ORCID de los autores y e-mail Josefina Cortés: https://orcid.org/0000-0001-6884-219X. E-mail: jfcortes@uc.cl Isabel Ugalde: https://orcid.org/0000-0002-5682-5357. E-mail: imugalde@uc.cl Julián Caviedes: https://orcid.org/0000-0002-8698-9504. E-mail: jjcavied@uc.cl José Tomás Ibarra: https://orcid.org/0000-0002-7705-3974. E-mail: jtibarra@uc.cl *Autor corresponsal

\begin{abstract}
Recibido: 29-04-2019. Aceptado: 28-08-2019. Fecha de publicación on-line: 26/09/2019
Citation / Cómo citar este artículo: Cortés, J., Ugalde, I., Caviedes, J., Tomás Ibarra, J. T. (2019). Semillas de montaña: recolección, usos y comercialización del piñón de la araucaria (Araucaria araucana) por comunidades Mapuche-Pewenche del sur de los Andes. Pirineos, 174, e048. https://doi.org/10.3989/pirineos.2019.174008

RESUMEN: La araucaria (Araucaria araucana), o pewen en lengua mapuche, es un árbol nativo de Chile y Argentina con fuerte importancia cultural para las comunidades mapuche-pewenche del sur de los Andes. Mediante el uso de metodologías cualitativas y técnicas etnográficas, se evaluaron los distintos usos actuales y los aspectos económicos y comerciales asociados al piñón, semilla de la araucaria, en una comuna cordillerana del centro-sur de Chile. Las prácticas locales relacionadas con el piñón dan cuenta de la importancia intrínseca y material de esta semilla, tanto para actores pewenche como para colonos. Nuestros resultados indican que estas prácticas incluyen desde la recolección, pasando por la venta a granel de las semillas, hasta la comercialización de productos elaborados (con valor añadido) de piñón y una creciente oferta de experiencias turísticas que integran piñones, araucarias, cultura y
\end{abstract}




\title{
• J. CORTÉS, I. UGALDE, J. CAVIEDES, J. T. IBARRA
}

paisajes locales. Concluimos que los procesos de recolección, uso y comercialización del piñón tienen dos componentes fundamentales que dialogan constantemente entre sí: un componente económico, en el sentido comercial y de subsistencia, y un componente biocultural, en el sentido espiritual, social, ecológico y alimentario.

PALABRAS CLAVE: Pueblos indígenas, bosque, productos forestales no madereros, comodificación, Chile.

\begin{abstract}
The monkey puzzle tree (Araucaria araucana), or pewen in Mapuche language, is a native tree from Chile and Argentina with a strong cultural importance for Mapuche-Pewenche communities from the southern Andes. Using qualitative methodologies and ethnografic techniques, we assessed the different current uses, and economic and commercial aspects associated with the seed of the monkey puzzle tree in a mountainous municipality of south-central Chile. Local practices related to the seed show the intrinsic and material importance of this seed for both Pewenche and other settlers. Our results show that these practices include from the gathering, through the bulk selling, to the commercialization of processed products from the seed and an increasing offer of touristic experiences integrating seeds, trees, culture and local landscapes. We conclude that gathering, uses and commercialization processes associated with the araucaria seed have two main components that establish a continuous dialogue: one economic component, in the commercial and livelihood vein, and a biocultural component, in the spiritual, social, ecological and food sense.
\end{abstract}

KEYWORDS: Indigenous peoples, forest, non-timber forest products, commodification, Chile.

\section{Introducción}

Naturaleza y cultura están indisolublemente relacionadas (Maffi, 2001). Desde un punto de vista fenomenológico, el mundo emerge con sus propiedades junto con la emergencia del observador en persona, en un contexto de acción práctica. Desde que una persona es un ser en el mundo, el proceso de venir-a-ser de ella es parte del proceso de venir-a-ser del mundo mismo (Ingold, 2000). De lo anterior emerge la importancia de las relaciones humano-naturaleza, pues estas relaciones configuran lo que significa el mundo. Y justamente en estas relaciones las personas tienen capacidad de agencia pues, en tanto que organismos vivos, ellas utilizan lo que necesitan del ambiente en cuanto les sea útil y/o funcional para sus vidas (Escobar, 1999). Si se comprende entonces a las personas como organismos relacionales, su capacidad de agencia no está solo supeditada al ámbito social, sino además es aplicable a través de la vida orgánica, pues los individuos en general necesitan de un otro para conformarse a sí mismos, pero también necesitan de otras cosas (e.g. recursos) para hacerlo (Ingold, 2000; Descola, 2013).

Sociedad y naturaleza están acopladas estructuralmente; aun cuando cada una está dotada de una lógica de funcionamiento autónoma, funcionalmente están compenetradas en la evolución e interacción cotidiana (Descola \& Gísli, 1996). El concepto "amasijo" (meshwork) sirve para dar cuenta de procesos socioambientales, pues existe una red de interacciones en las que se infiltran aspectos materiales y subjetivos, y cuya integración adquiere formas simbólicas que condicionan la práctica de los sujetos que habitan un territorio en particular (Skewes \& Guerra, 2016).

En esta línea resulta fundamental entender el papel que tienen los árboles, con sus frutos y semillas, en la configuración de los paisajes locales. Los árboles han sido compañeros permanentes de los seres humanos. La reflexión acerca de la mutua constitución de poblaciones humanas en relación con especies bioculturales clave (sensu Garibaldi \& Turner, 2004; Ibarra et al., 2012; Jacques-Coper et al., 2019) merece ser profundizada, especialmente en lo relativo a los árboles (Kohn, 2013; Skewes \& Guerra, 2016). La apropiación humana de los árboles y sus productos, y sus efectos sobre el ambiente y la vida cotidiana de quienes utilizan estos recursos, no dependen solo de las características de éstos, las técnicas y los ciclos de extracción, comercialización y comodificación, sino también de los procesos sociales relacionados a ello (Otero, 2006; Donoso et al., 2014).

Los bosques de araucaria (Araucaria araucana), o pewen en lengua mapuche, son únicos a escala planetaria al tratarse de un sistema dominado por un árbol considerado un verdadero "fósil viviente" (Gedda, 2010). La araucaria, una especie clasificada en Peligro de Extinción a nivel mundial (Premoli et al., 2013), es un árbol que alcanza hasta los treinta metros de altura y puede envejecer más de 1.000 años (Aguilera-Betti et al., 2017). La importancia de la araucaria no solo radicaría en su valoración alimenticia, botánica y ecológica (Ibarra et al., 2010; Barreau et al., 2016; Cockle et al., 2019), sino también en su importancia cultural para el pueblo mapuche-pewenche (pewen: araucaria y che: gente; pewenche = gente del pewen) (Herrmann, 2005, 2006; Gedda, 2010). Los pewenche son los mapuche que habitan las tierras altas de la cordillera de los Andes del centro-sur de Chile, donde co-habitan con la araucaria.

Escritos y crónicas dan cuenta de la importancia del piñón como recurso de subsistencia para los pewenche (Wilhelm de Mösbach, 1992; Coña, 2010). La valoración cultural de las araucarias no sólo radicaría en el árbol como tal, sino también en los piñones (semilla de la araucaria) y lo que ellos significan más allá del aspecto econó- 
mico (Reiche, 2013). El piñón tiende a ser recolectado con motivo de consumo alimenticio y de intercambio comercial (Herrmann, 2005; Perasso, 2012). También, la recolección y consumo del piñón podrían tener una importancia creciente en el turismo de naturaleza y en lo que algunos autores han llamado como "etno-turismo" (Gedda, 2010). Sin embargo, las posibilidades económicas contemporáneas basadas en el piñón varían ampliamente entre comunidades, debido básicamente a las posibilidades de acceso a los bosques y al mercado por parte de las comunidades (Isla, 2001; Barreau et al., 2016). Aunque se ha señalado la importancia del piñón a distintos niveles, aún escasean trabajos que profundicen en la importancia económica actual del piñón y los alcances culturales del uso comercial de esta semilla (e.g. procesos de comodificación de ella). Un estudio de estas características es particularmente necesario en el territorio ancestral del pueblo Mapuche, o Wallmapu en mapuzugun (i.e. lengua mapuche), el cual también es habitado por residentes de múltiples orígenes (e.g. colonos), quienes igualmente utilizan la semilla de esta especie.

El objetivo de este trabajo fue describir y analizar los usos culturales, en particular los aspectos económicos asociados al uso del piñón por parte de los habitantes pewenche y colonos del centro-sur de Chile. En concreto, (i) identificamos los principales usos actuales del piñón; (ii) describimos las distintas formas de comercialización del piñón; y (iii) presentamos la valorización que le dan al piñón los habitantes pewenche y colonos de Lonquimay, una comuna cordillerana del centro-sur de los Andes de Chile.

\section{Metodología}

\section{1. Área de estudio y contexto etnográfico}

La Región de La Araucanía se encuentra en la zona centro-sur de Chile (Figura 1), extendiéndose desde los $37^{\circ} 35^{\prime} \mathrm{S}$ hasta $\operatorname{los} 39^{\circ} 37^{\prime} \mathrm{S}$. Esta región tiene una superficie de $31.842 \mathrm{~km}^{2}$, representando un $4,2 \%$ del territorio nacional chileno y un total de 957.224 habitantes (INE, 2017). Administrativamente, la región se divide en dos provincias; Malleco por el norte y Cautín por el sur. Este estudio se realizó en la Comuna de Lonquimay, específicamente en los sectores de Quinquén, Lonquimay centrourbano, Icalma y Cruzaco (Figura 1). La Comuna de Lonquimay se encuentra en la parte oriental de la Provincia de Malleco, tiene una superficie total de $3.914 \mathrm{~km}^{2}$ y 10.251 habitantes, de los cuales el $63,1 \%$ es población rural y el $56 \%$ se auto identifica como mapuche (INE, 2017). La Región de La Araucanía es la región más pobre de Chile, y Lonquimay, por su parte, es la segunda comuna más pobre de la región con un $64,4 \%$ de habitantes en situación de pobreza (CASEN, 2017). Debido a las fuertes pendientes y la baja calidad de sus suelos, lo que dificulta el desarrollo de la agricultura, las principales actividades económicas comunales son la actividad forestal, la ganadería y los productos relacionados con la recolección del piñón. Sin embargo, en el último tiempo, ha surgido con mayor fuerza la actividad turística, teniendo en consideración las bellezas naturales de la comuna (Plan de Desarrollo Comunal Lonquimay, 2016). La comuna se ubica también dentro de la Reserva de la Biosfera Araucarias, dada la gran presencia de bosque nativo, concretamente del tipo forestal araucaria (Gajardo, 1993).

\subsection{Trabajo de campo}

La metodología implementada es de carácter cualitativa, utilizando técnicas etnográficas. Durante tres meses (septiembre-noviembre de 2017) se residió en territorio habitado por comunidades pewenche y no pewenche (colonos) de las zonas urbanas y rurales de Lonquimay. Utilizamos tres herramientas de recopilación de información. En primer lugar, se realizaron 25 entrevistas semi-estructuradas a actores del territorio que estuvieran relacionados con el piñón. Estos actores fueron identificados mediante la aplicación de la metodología de selección por "bola de nieve" (Bernard, 2005). También implementamos una entrevista grupal o "grupo focal", con participación de seis personas, la cual se diseñó de manera tal que se pudiera recolectar información de un colectivo social que se conocía previamente y que mantenía una relación activa con el piñón y la araucaria (Sandoval, 2002). Antes de cada entrevista obtuvimos la aprobación de un consentimiento informado libre, cumpliendo con las exigencias del Comité de Ética de la Pontificia Universidad Católica de Chile.

Finalmente, realizamos observación participativa. Esta aproximación nos permitió vivir en el territorio y acceder a vínculos y niveles de comprensión de los relatos y del fenómeno que hubiesen sido imposibles de alcanzar desde un levantamiento de información durante periodos cortos y/o basados sólo en entrevistas. La observación participativa fue registrada mediante notas de campo y fotografías, donde recopilamos experiencias concretas asociadas a vivir en la pensión de una familia lonquimayina; participar de actividades locales (i.e. ferias, celebraciones, etc.) y ceremonias de origen mapuche (Trafkintu, Wetripantu, Trawün); desarrollar entrevistas informales; compartir alrededor del fogón; asistir a talleres con funcionarios públicos de la comuna; participar de asados de cordero y traslados con funcionarios del Programa de Desarrollo Territorial Indígena (PDTI); trabajar en la biblioteca pública, en la plaza de Lonquimay y en la delegación municipal de Icalma (para poder tener acceso WIFI); visitar a las personas en sus hogares; compartir largas mateadas (muy dulces y también amargas); junto con varias otras actividades.

\subsection{Análisis}

El análisis de la información recogida en las entrevistas, grupo focal y observación participativa se realizó a través del software de análisis cualitativo MAXQDA 


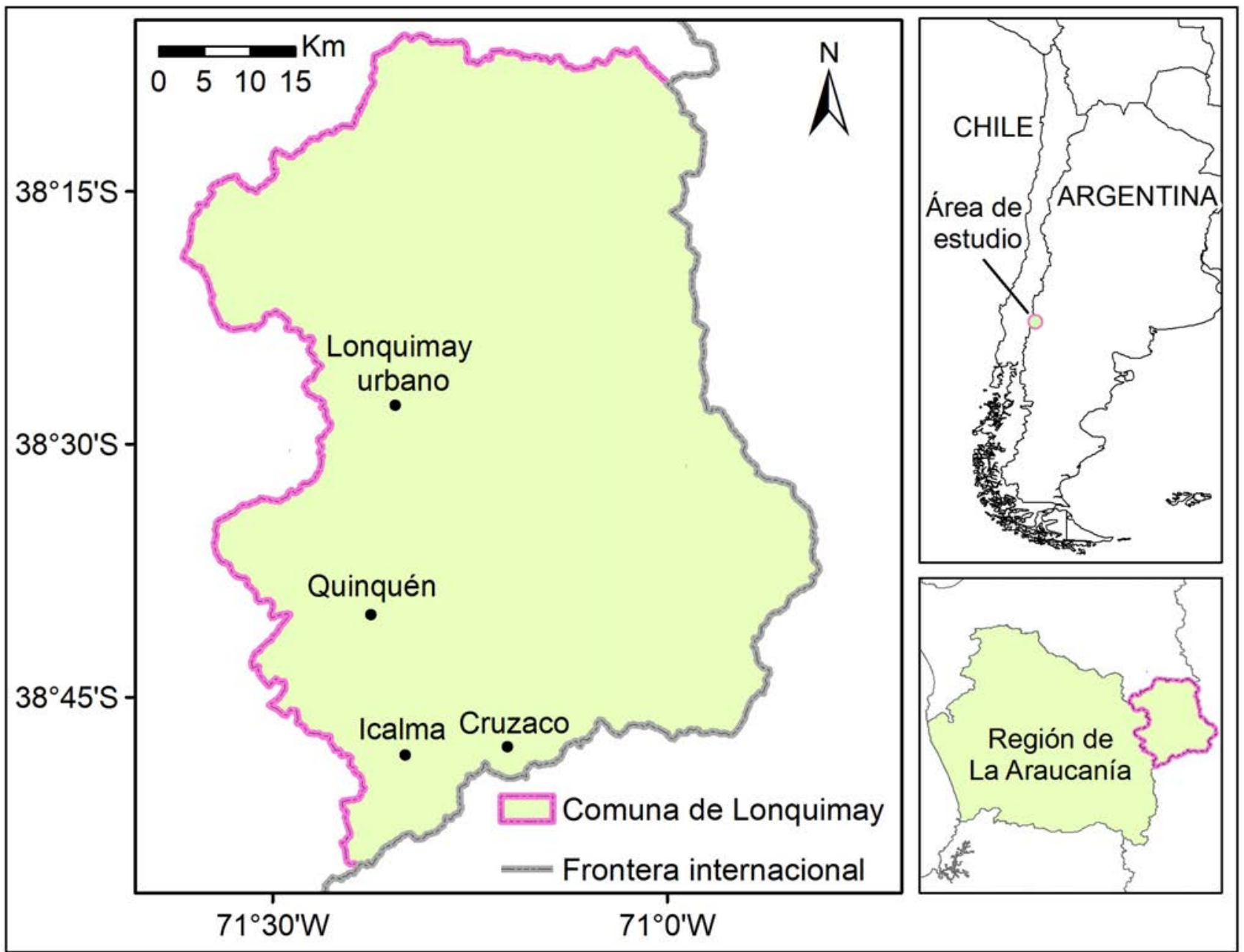

Figura 1. Área de estudio en la Comuna de Lonquimay en la Región de La Araucanía al sur de los Andes, Chile. Se indican las localidades específicas en donde se trabajó: Quinquén, Lonquimay centro-urbano, Icalma y Cruzaco.

Figure 1. Study area in the Municipality of Lonquimay in the La Araucania Region in the southern Andes, Chile. We indicate the specific localities where fieldwork was conducted: Quinquén, Lonquimay urban-center, Icalma and Cruzaco.

Analytic PRO-12. Con este software se sistematizó la información contenida en los relatos a través de una codificación abierta. Posteriormente, estos códigos fueron agrupados en categorías, que respondieron a su vez a categorías de carácter más analítico y reflexivo, dando origen a un árbol de códigos o categorías conceptuales. Así también, este software permitió anclar la información de carácter visual, ya sean fotografías y material audiovisual, a la información ya sistematizada en categorías o nodos, a través de la utilización de "Memos" y "Anotaciones" que entrega la plataforma.

\section{Resultados y discusión}

Al llegar a la Comuna de Lonquimay es posible visibilizar, a lo lejos, en las cumbres de los cerros aledaños, los bosques de araucarias o pewen, los que "son los últimos habitantes de la cordillera" (Mujer no pewenche). Al hablar del pewen, la gente de la Comuna transmite cómo la araucaria y su semilla eran elementos claves de la naturaleza con una preponderancia sociocultural y económica vital en Lonquimay. Podríamos asegurar que la totalidad de los habitantes de la Comuna, tanto mapuche-pewenche como colonos, han recolectado o recolectan, comieron o comen, y utilizan o alguna vez utilizaron el piñón. Así, pewenche y colonos comparten un mismo territorio que los liga en torno a la semilla de la araucaria tanto en su valor cultural como en sus distintos usos.

\subsection{El piñón a granel}

La venta del piñón a granel, en saco y sin procesar, es el reflejo del valor de cambio que tanto habitantes colonos como pewenche le atribuyen al piñón. Esta práctica, la más utilizada por los habitantes de Lonquimay, se basa en la venta de grandes cantidades de piñones "al por ma- 
yor" a pequeños o medianos comerciantes -el intermediario-, que luego re-venden a comerciantes mayores (ferias urbanas, supermercados, etc.). Una familia conformada por tres adultos puede llegar a recolectar un saco de 65 a $85 \mathrm{~kg}$ en un día y, en un año de buena producción, hasta 32 sacos, es decir, $2.500 \mathrm{~kg}$ aproximadamente por temporada (Neira, 1995). Las comunidades y familias recolectoras del piñón, o nguilliu en mapuzugun, son el primer eslabón de una larga cadena de comercialización. Los recolectores, quienes hacen el trabajo más pesado, son quienes venden a menor precio y quienes consiguen los menores réditos de esta actividad económica. Los precios de venta del piñón a granel se definen en base a dos grandes criterios: (i) la disponibilidad y fecha de recolección y (ii) las necesidades inmediatas de cada vendedor. Tacón \& Palma (2006) plantean que "al inicio de la temporada cada piñonero establece una banda de precios tentativa para su producto, dentro de la cual se realizará la negociación". Sin embargo, nosotros nos percatamos que en la práctica no suele ser así y que el intermediario es quien, jugando con estos dos criterios, establece el precio de venta de ese año.

La disponibilidad anual del piñón varía entre años (Donoso, 2006). Así, hay años en que la disponibilidad de piñón es alta y años en los que se vuelve "un privilegio poder consumirlos" (Mujer recolectora pewenche). Esta condición es la que comuneros y colonos definen como "añera". Ello implica que los precios de venta del piñón sean inestables y fluctúen de un año a otro, siendo mayores los precios en años de poco piñón y menores en años de abundancia (Figura 2).

La recolección del piñón se hace en épocas estivales y las fechas exactas varían según el año y la cantidad de semillas. Por lo general, se hace a partir del 15 de febrero hasta fines de marzo, época que, según un participante "coincide con un montón de gastos con los que tienen que lidiar las familias del sector; es cuando los niños tienen que entrar al colegio, tienen que comprar los útiles, tienen que juntar alimento y justo no hay otro tipo de traba- jo" (Hombre recolector pewenche). De esta manera, la venta del piñón se da en un contexto de escasez, lo que hace que esta semilla sea vista como un medio para satisfacer necesidades a corto plazo. Este mecanismo hace que quien más lo necesite lo vende más barato, dando de esta forma inicio a la negociación de la venta y precios de ese año. "A veces la cuenta cae bien, pero otras veces cae mal, porque todos vienen con un precio calladitos no más: yo pago tanto, el otro paga menos y asi se van haciendo competencias. Usted a veces entrega a 500 pesos, mañana viene uno a 700 y yo entregué ayer a 500 pesos. Así es el precio del piñón, es variable" (Colona recolectora).

\subsection{El valor agregado: diversificación en base al piñón}

La elaboración de productos gastronómicos a partir del procesamiento del piñón se concibe como una estrategia de venta y consumo a largo plazo. Si bien este sistema de comercialización del piñón tendría un "potencial agroindustrial" (FIA, 2010), en la práctica persiste un modo artesanal de preparación donde "quien participa es la familia completa; cada familia ocupa su cocina para deshidratar" (Productora pewenche). El tostado, pelado y deshidratado se hacen "a mano" y en las propias cocinas de las familias. "Yo creo que eso es lo más lindo de todo eso. Aquí participa desde el más chico hasta el más adulto. La producción es como la recolección" (Productor pewenche refiriéndose al papel de la familia en el proceso).

Los productos elaborados de piñón son cada vez más comercializados y, pese a que son una fuente importante de ingresos para quienes los producen, los procesos asociados en la elaboración siguen teniendo una fuerte implicación cultural en las familias. Para fabricar productos elaborados (e.g. harina), el piñón debe pasar por varios procesos $y$, por lo tanto, por varias manos porque el piñón se debe tostar (o cocer), pelar y moler para hacer harina. Estos procesos en torno a la cocina fortalecen los lazos de parentesco entre los recolectores, ya que gran parte del nú-


Figura 2. Diferencia de precios entre (A) kilogramo de piñón a granel y (B) un kilogramo de harina de piñón, según año bueno y año malo de producción. Los precios corresponden al valor promedio señalado por los participantes y diario de campo.

Figure 2. Difference in prices between a (A) kilogram of bulk sale of seeds and (B) kilogram of flour from seeds of the monkey puzzle tree (piñón), according to a "good" and "bad" production year. The prices correspond to the average amount reported by the participants and field diary. 
cleo familiar participa activamente en alguna de las etapas del proceso. De esta forma, al reunir a la unidad familiar en torno a la cocina, se fortalecen afectos, costumbres y experiencias. Más aún, esta instancia se transforma en un mecanismo de transmisión del conocimiento, tanto vertical (hacia los niños y jóvenes) como horizontal (entre familiares de la misma generación) (Barreau, 2014).

Al preguntarle a un productor pewenche qué hacía con los piñones que le compraba a los recolectores, él relataba: "todos esos piñones después los mando a pelar, entonces a la misma familia que me entrega (vende) piñón, se le retornan los sacos y en el invierno me trabajan pelando el piñón”. En torno a la producción se ha desarrollado una estructura de división del trabajo, donde familias completas se dedican a la recolección en verano, venta en otoño y al mismo tiempo tienen trabajo en invierno. Esta diferenciación del trabajo implica, frecuentemente, colaboración recíproca entre distintas familias en la búsqueda de que el proceso resulte ventajoso para todos y, en muchos casos, utiliza estructuras propias de una cooperativa.

\subsection{La economía familiar más allá de lo local}

Históricamente, y aún en la actualidad, las comunidades recolectoras planificaban sus reservas de piñón para ser consumidas a lo largo de todo el año. Esta semilla, mediante diferentes técnicas de guardado, se podía conservar desde su colecta y ser utilizada en distintas preparaciones durante el año (e.g. harina, chuchoca, locro). Esta tradición, junto con procesos asociados a la diversificación que han desarrollado productores y consumidores, dio origen a que los productos elaborados a partir del piñón se abrieran a otros mercados y dejaran de ser considerados exclusivamente para el autoconsumo familiar, sino también como una promisoria actividad económica. Estos factores han sido determinantes en la profundización de las transacciones de mercado dentro del ciclo de reproducción de la práctica cultural de recolectar y usar piñones, es decir, en el proceso de comodificación del sistema del piñón (Friedmann, 1980). Cabe destacar que la opción consumo y producción/venta no han sido excluyentes. Todas las personas que vendían productos elaborados basados en el piñón indicaron que éste seguía siendo una parte importante de su dieta familiar durante el año.

La venta de sopaipillas con harina de piñón, por ejemplo, es una de las tantas formas que los habitantes de la comuna han desarrollado para darle un valor agregado al piñón. Durante nuestra inspección etnográfica notamos que productos derivados del piñón, tales como galletas, tartas, puré, cerveza y café, entre otros, han nacido de la mezcla de un conocimiento local, por un lado, y de un conocimiento formal de agentes externos, por otro (talleres impartidos por la municipalidad, universidades, etc) (Tabla 1; Figura 3). El proceso de comodificación del piñón ha estado relacionado con un creciente interés por consumidores de grandes ciudades de Chile e incluso del extranjero. Muchos de los productos que se elaboran actualmente en la comuna no se quedan allí mismo, sino que recorren largas distancias para poder ser consumidos $\mathrm{y}$, por lo tanto, también elevan su valor. Al preguntarles por sus principales compradores, un productor pewenche nos contesta: "Temuco, Concepción, Santiago... en Santiago es el mayor despacho que hago". Los sitios de venta de los productos en estas grandes ciudades son frecuentemente "tiendas especializadas" o "tiendas gourmet", las cuales tienen una connotación gastronómica distinta a las

Tabla 1. Distintas preparaciones basadas en el piñón de la araucaria por familias pewenche y colonas de la Comuna de Lonquimay, sur de los Andes.

Table 1. Different preparations, from the seed of the monkey puzzle tree, by Pewenche families and settlers from the Municipality of Lonquimay, southern Andes.

\begin{tabular}{|l|l|}
\hline \multicolumn{1}{|c|}{ Preparaciones tradicionales } & Nuevas preparaciones \\
\hline Harina & Galletones \\
\hline Ensalada con piñón & Galletas \\
\hline Catutos & Alfajores \\
\hline Sopaipillas & Tartas \\
\hline Chuchoca & Mermeladas \\
\hline Locro & Licores \\
\hline Piñones cocidos & Budín \\
\hline Piñones salteados & Cerveza \\
\hline Piñones tostados & Puré \\
\hline La salsa (puré) & Cuscús de piñón \\
\hline Muday & \\
\hline Café & \\
\hline
\end{tabular}



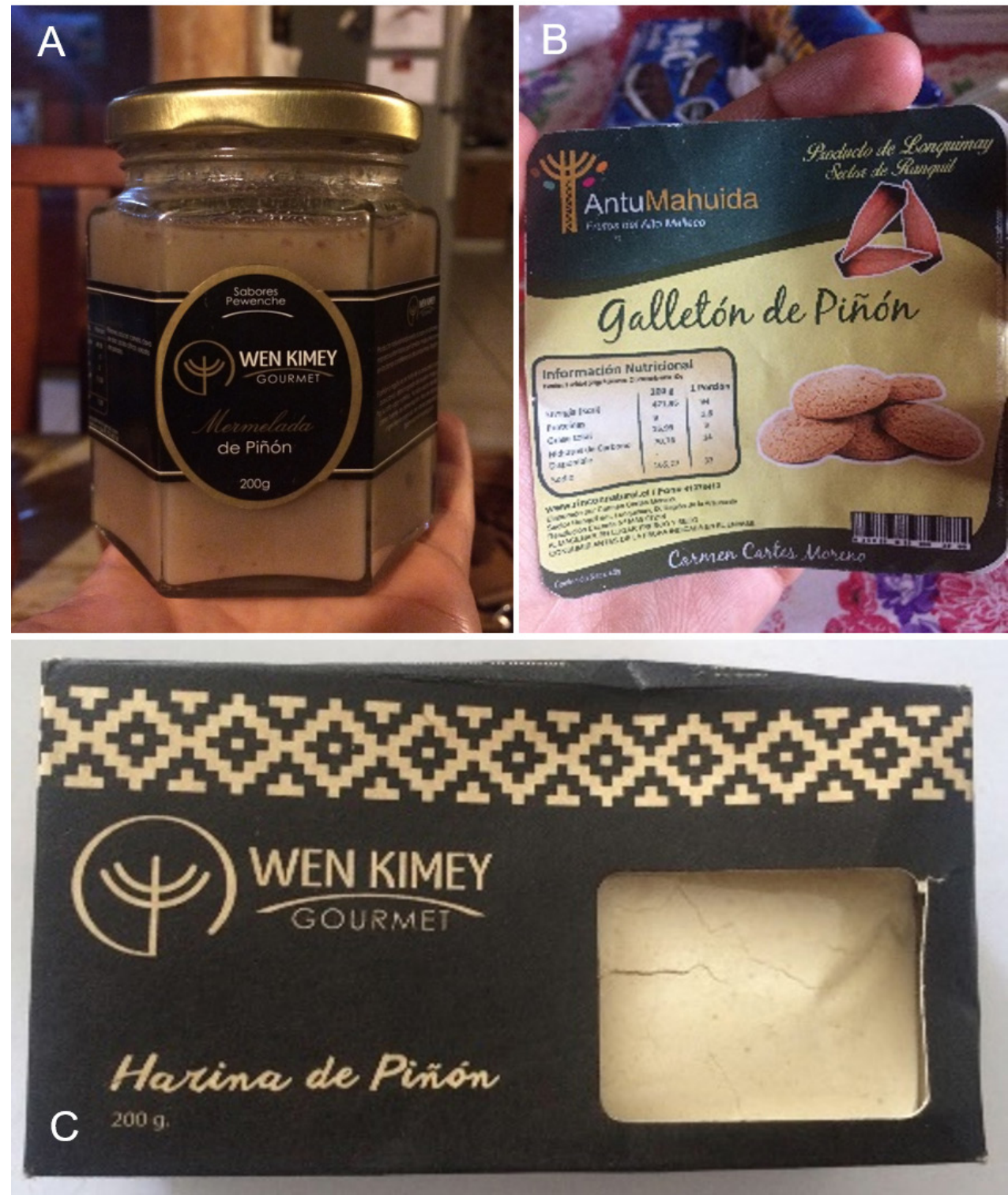

Figura 3. Distintas preparaciones basadas en el piñón: (A) mermelada, (B) galletones y (C) harina. Fotos: A, C: Josefina Cortés. B: Isabel Ugalde.

Figure 3. Different preparations from the seed of the monkey puzzle tree (piñón): (A) jam, (B) biscuits, and (C) flour. Photos: A, C: Josefina Cortés. B: Isabel Ugalde.

de una feria local y, por lo tanto, son tiendas con un tipo de cliente particular y dispuesto a pagar altos precios.

\subsection{Experiencias con "sabor a piñón": turismo y gastronomía}

Aventurarse a vivir experiencias nuevas, en las que se encuentren dos culturas, sobre todo en lo culinario, está ligado con el turismo. El turismo va en ascenso en Lonquimay, al igual que el desarrollo económico del piñón. La gastronomía basada en el piñón se posiciona como un elemento fuerte dentro del desarrollo turístico de la co- muna: "hartas cosas que hacemos con piñón están relacionadas con el tema de turismo" (Operador turístico pewenche en Icalma). Además de contarnos que el turismo es una buena fuente económica, el operador turístico pewenche fue enfático en plantear la importancia de dar a conocer la historia local: "como que de ahi nace todo esto. El turismo para mí es una tremenda herramienta, para darle valor a mi pueblo mapuche”. Él y otros operadores turísticos nos relatan la importancia de rescatar y visibilizar su cultura mediante la cocina y el relato: "donde le contamos una historia, donde lo invitamos a ser parte de la comunidad, como a vivir una experiencia” 
(Operador turístico pewenche). "Se hacen tour a los pinos" nos cuenta un colono como, por ejemplo, el caso del sendero "Kume ad Mawida" y "Kelutrufken" dentro de la comunidad de Quinquén, donde el turista puede conocer las "veranadas" (i.e. práctica tradicional de traslado del ganado a las zonas altas de la cordillera en el periodo estival) y convivir con bosques de araucarias.

La reflexión desde los mismos habitantes es a desarrollar un turismo local que beneficie a su gente. Una operadora turística pewenche reflexiona acerca de esto: "Se podría valorar más lo que tenemos... Si esto parte porque nosotros le hemos perdido harto el valor a nuestro pewen. Y por los niños también, que crezcan con esa visión, valorando lo que tienen ellos; la araucaria, el piñón... Porque es algo que no cualquiera lo tiene. Y nosotros lo tenemos y no lo valoramos". Para el desarrollo local alrededor de las araucarias y el piñón es fundamental la colaboración y asociación entre quienes realizan turismo, ya sea tengan cabañas, realizan tours u ofrezcan comidas. "Se va pasando el dato" nos cuentan, y por ejemplo, "si el turista quiere comer algo de piñón se le dice que vaya a comer para allá" (Mujer pewenche en un local con venta de comida en base a piñón).

Un aspecto importante que ha contribuido a la venta de piñón es justamente el aumento de las instancias de venta en los que se pueden ofrecer estos productos elaborados. Un ejemplo de éstas son las fiestas locales de verano que se desarrollan en la comuna de Lonquimay; el “Asado de Chivo" y la "Fiesta del Piñón". Son estos espacios de encuentro donde la gente de la comuna tiene la posibilidad de dar a conocer sus productos y vender. Las distintas ferias gastronómicas locales también son una instancia para mostrarse.

La multiplicación de ferias gastronómicas como forma de dar a conocer productos alimentarios locales cristaliza una especie de sincretismo entre el conocimiento local y el de los visitantes. En estas instancias ocurre un punto importante en el desarrollo comercial, visibilización y valorización del piñón, los bosques de araucaria y el pueblo mapuche-pewenche. En estas ferias gastronómicas es donde los habitantes de Lonquimay han participado con algunos productos de piñón y han abierto las puertas a la venta de piñón fuera de lo local, el "salir a darse a conocer". Este salir a darse a conocer también tiene que ver con invitar al externo, al turista, a visitar la comuna y a vivir una "experiencia distinta". La existencia de restaurantes, emprendimientos locales, hosterías con ofertas gastronómicas basadas en el piñón, una cervecería que cocina a partir de café de piñón, entre otras, se posicionan como un elemento de esta experiencia distinta que nos cuentan: "la experiencia gastronómica en torno al piñón". Lo anterior visibiliza la importancia que nos enfatizaron sobre desarrollar el turismo en esta área, sobre todo, con pertinencia local mapuche-pewenche: "ellos (el turista) tienen que sentir el sabor que sea de piñón, porque ellos siempre dicen de que ellos van a otras partes y como que no le encuentran el sabor a piñón... como por ejemplo el alfajor lleva un poquito de licor, lleva coñac, un poquito, entonces muchas veces ellos le encuentran más sabor al coñac (risas...)" (Cocinera y operadora turística pewenche).

La mezcla de productos locales con preparaciones nacionales es el reflejo de una gastronomía nacional que incorpora diversidad de identidades y que colabora en la construcción de una identidad nacional nueva (Aguilera, 2016). Este fenómeno es una forma efectiva de incorporar nuevas preparaciones, junto con visibilizar y valorar lo local, combinando "lo conocido con lo desconocido" en preparaciones como puré de piñón, cerveza de piñón, mermelada de piñón, cuscús de piñón, puré de piñón, entre sus principales.

\subsection{La recolección no es solo recoger}

Todo el trabajo que hay detrás de crear un producto de piñón implica, como se explicó anteriormente, a los bosques de pewen, a la participación de familias, a la fauna (e.g. la cachaña Enicognathus ferrugineus para dispersar la semilla [Speziale et al., 2018]), al viento, a la lluvia y al sol, entre otros elementos fundamentales. Esta es una maraña interconectada de procesos vitales asociados los unos a los otros (Skewes \& Guerra, 2016). Aunque lo menos dañino para la araucaria, en términos tanto ecológicos como espirituales, de acuerdo con los participantes, es re-

Tabla 2. Tipos de piñón, características asociadas a la recolección y usos en la Comuna de Lonquimay, sur de los Andes de Chile. Table 2. Types of seeds of the monkey puzzle tree, characteristics associated with the gathering and uses in the Municipality of Lonquimay, southern Andes of Chile.

\begin{tabular}{|l|l|l|l|}
\hline \multicolumn{1}{|c|}{ Tipo (nombre local) } & \multicolumn{1}{|c|}{ Época de recolección } & \multicolumn{1}{|c|}{ Uso y descripción } & \multicolumn{1}{c|}{ Modo de recolección } \\
\hline Puyen* & $\begin{array}{l}\text { Finales de diciembre } \\
\text { hasta principios de } \\
\text { febrero. }\end{array}$ & $\begin{array}{l}\text { Por lo general para hacer harina y muday. } \\
\text { Son lechosos y blandos. Se cosechan por } \\
\text { necesidad. }\end{array}$ & $\begin{array}{l}\text { Se sube a la araucaria o } \\
\text { se “lacean" para botar las } \\
\text { cabezas. }\end{array}$ \\
\hline Yaten & $\begin{array}{l}\text { Finales de febrero hasta } \\
\text { principios de mayo. }\end{array}$ & $\begin{array}{l}\text { Se utilizan para chuchoca, harina y } \\
\text { cocción. Son los primerizos, más dulces y } \\
\text { ricos. }\end{array}$ & $\begin{array}{l}\text { Se recogen del suelo, ya } \\
\text { que caen por si solos de la } \\
\text { araucaria }\end{array}$ \\
\hline Guillín & $\begin{array}{l}\text { Inicio del derretimiento } \\
\text { de la nieve (septiembre a } \\
\text { noviembre) }\end{array}$ & $\begin{array}{l}\text { Se usan de inmediato al ser fáciles de } \\
\text { moler. }\end{array}$ & $\begin{array}{l}\text { Se recogen del suelo, cuando } \\
\text { se va la nieve. }\end{array}$ \\
\hline
\end{tabular}

* Este tipo es el de menor valor comercial. 



Figura 4. Distintos componentes y procesos asociados a la recolección y uso de piñones de araucaria: (A) cabeza de piñón en árbol, (B) cabeza de piñón abierta, (C) lonko (autoridad) pewenche piñoneando (recolectando) y (D) piñones salteados. Fotos: A, B, D: Manuel Gedda. C: José Tomás Ibarra.

Figure 4. Different components and processes asssociated with the gathering and use of seeds of the monkey puzzle tree: (A) cone, (B) opened cone, (C) pewenche lonko (community chief) gathering seeds, and (D) sautéed seeds. Photos: A, B, D: Manuel Gedda. C: José Tomás Ibarra.

colectar los piñones desde el suelo, en la práctica aquello varía en función de la época del año (Tabla 2; Figura 4). Los modos de piñonear van desde recolectar desde el suelo luego de que la cabeza de piñón "reviente sola y esperar a que caigan nomás con el viento", hasta apurar este proceso "laceando" (tirando un lazo) hacia las cabezas de piñón para que éstas boten la semilla. También hay quienes trepan por el tronco del árbol, para acercarse a las cabezas o conos de piñón, y así moverlos para que los boten. Nos comentan también, tanto colonos como pewenche, que algunos cortan los ganchos para vender las cabezas de piñón verde. Esto último, según los participantes, es fuertemente sancionado entre los mismos habitantes, quienes entienden el daño causado porque ven los efectos posteriores: "cuando bota la cabeza, la arranca, no vuelve a salir nunca más, entonces uno mismo va matando la araucaria". Una recolectora pewenche agrega: "Igual la gente los saca antes po... Saca las cabezas enteras, verdes. Eso igual está prohibido po, no debería ser, pero hay gente que lo hace..." Estas prácticas fueron frecuentemente senaladas, por los propios participantes, como no sustentables para la mantención y regeneración de la araucaria.

La regeneración de la araucaria estaría siendo afectada por múltiples factores, incluyendo los incendios foresta- les, explotación maderera (en el pasado), crisis climática, la introducción de especies invasoras, la fragmentación de su hábitat y enfermedades, entre otras causas (Aagesen, 1998; Pauchard \& Alaback, 2004; González et al., 2005; Zamorano-Elgueta et al., 2012; Molina et al., 2015; Besoain et al., 2019). Una eventual sobre-cosecha del piñón podría estar ocurriendo en distintas localidades, lo que se asociaría a la creciente demanda existente más allá de los límites comunales, regionales e incluso nacionales. Aunque esta eventual sobre-cosecha fue reconocida por algunos participantes, aún no ha sido evaluada empíricamente. Incluso, en período de cosecha, es posible ver a numerosos actores externos a las comunidades que están cosechando piñones sin regulación. El proceso de comodificación del piñón ha acarreado una fuerte demanda de la semilla a granel en supermercados nacionales y extranjeros, con consecuencias sobre la economía y prácticas de recolección, uso y comercialización.

La escasez, la pobreza, la necesidad de comer, vender $\mathrm{y}$ vivir permea la forma de acceder al bosque y recolectar. Respecto al piñón, el sentido práctico-comercial está en un constante proceso de negociación con un sentido simbólico-ritual en la recolección (Isla, 2001): "pero ahí la gente era muy respetuosa, porque hablaban con la arau- 
caria, le pedían permiso que le iban a bajar piñones. Ahora nosotros... Eso es lo que se ha perdido" (Recolectora pewenche). Los habitantes y recolectores son conscientes de esto. Existe una determinación y necesidad de rescatar el valor biocultural del pewen y el piñón, y usarlo a su vez "como un enganche comercial", como nos decía un pewenche. Él mismo explicitaba: "pensar que con una bolsita de harina de piñón sale un tema de conversación, entonces más que venderle es un enganche".

Resulta indispensable dar cuenta que la valorización y visibilidad del piñón -en sus distintas formas-, tanto la externa como la interna de la comuna, debe también, supeditarse a lo complejo y sacrificado que resulta recolectar el piñón. El acceso al monte -el bosque- que implica muchas veces caminar hacia zonas altas y climáticamente extremas, el sacrificio físico que requiere estar agachado recogiendo uno a uno los piñones y "sacándoles la colita", llevar al hombro sacos de piñón, y el desplazamiento -hoy en día con mayor posibilidad de subir en camioneta-, son factores indispensables de considerar en todo lo que implica tener en las manos una galleta de piñón: "Todo lo que cuesta, porque es un tremendo sacrificio ir a recolectar los piñones. Imagínese salir todos los días a las seis de la mañana de su rancho, y ahí uno está todo el día afuera, está todo el día ahí hasta que hace su carga. Y eso no lo haces aquí al lado de tu rancho, sino que lo haces en la punta del cerro y de ahi tienes que bajar con tu carga al hombro" (Hombre pewenche). Una recolectora colona, concuerda: "Y lo otro que dicen es que no puede valer el piñón más que el trigo... Pero el piñón es más sacrificado, porque usted tiene que andar por las montañas, por los cerros, con piñones al hombro" (Mujer colona).

$\mathrm{Si}$ bien fuimos testigos de que muchos habitantes tienen las araucarias literalmente en sus patios, la acción misma de recolección requiere de tiempo, del uso del cuerpo, de fuerza, de ocupar las manos; "salen hasta callos". La recolección, de modo artesanal, la realizan familias completas, niños, abuelos, padres y madres. Generalmente recolectan una parte para su consumo, pero también se destinará una parte para la venta o intercambio. Bajo esta realidad, la unidad de producción no es distinta de la unidad de consumo, por lo que la actividad doméstica de preparación no se separa de la actividad productiva (Schejtman, 1980). Comprender y valorar lo anterior es fundamental, sobre todo en territorios rurales, lugares en los que se extraen hierbas, frutos, hongos, semillas, que llegan a las mesas de familias locales, pero también a grandes ciudades de Chile y el extranjero.

\section{Consideraciones finales}

Tras una aproximación cualitativa, con la utilización de técnicas etnográficas en la Comuna de Lonquimay, concluimos que la recolección, uso y comercialización del piñón se posicionan como prácticas con importante valor cultural y económico, tanto para colonos como para pewenche de la zona. Estas actividades son parte de una forma de vida y una vinculación con el territorio que emerge en la cotidianeidad, desde una semilla icónica del sur de los Andes hasta los múltiples sitios, rurales y urbanos, en donde se consume el piñón en la actualidad. En ese sentido, es posible plantear que el valor y uso económico asociado al piñón no tiene exclusividades ni distinciones de origen mapuche-pewenche dentro de las localidades en las que pudimos trabajar.

A nivel global, es posible establecer paralelismos entre el sistema de recolección, uso y comercialización del piñón con otros productos forestales no madereros que se están enfrentando a procesos similares de comodificación. Un ejemplo de ello es la quinoa y su posicionamiento como cultivo comercial, el cual se inició entre los consumidores de los países industrializados y, posteriormente, en los mercados internos de los países en donde el cultivo es nativo (Neri, 2017). Este posicionamiento ha significado una alteración en las relaciones sociales y de trabajo que rodean la producción de quinoa. Con el desarrollo acelerado de un mercado de exportación y el aumento en las áreas donde se cultiva la quinoa, las prácticas de reciprocidad, en las que los campesinos dependían principalmente del intercambio informal de mano de obra con vecinos y familias para administrar la cosecha, se volvieron cada vez más escasas (Kerssen, 2015). Otro ejemplo relevante a nivel internacional es el estudio del proceso de producción de la caña de azúcar, desde su cosecha hasta su comercialización y consumo como azúcar granulada (Mintz, 1996). Con ciertas similitudes al proceso por el que está transitando el piñón de la araucaria y la quinoa, la historia de cómo los ingleses se convirtieron en consumidores de azúcar muestra como se relaciona la comida, desde lo más íntimo de las cocinas y la familia como agente, con procesos multiescalares de comodificación. Cronistas, historiadores e investigadores (Bengoa \& Valenzuela, 1983; Bengoa, 2000; Isla, 2001; Tacón \& Palma, 2006; Domeyko, 2010; Perasso, 2012; Reis et al., 2014) convergen en que la forma de apropiación por excelencia del piñón fue y es económica, haciendo uso de éste como fruto comestible. Sin embargo, para el caso del piñón, la relación comida-comodificación refleja que ésta relación se construye y deconstruye ante la aparición de nuevos agentes de cambio socioambiental (e.g. turismo y mercados distantes).

La forma en cómo los sujetos son capaces de transformar la naturaleza y convertirla en alimento, apareciendo imaginarios, creencias y representaciones, responde a las formas en cómo se apropian de la naturaleza (Aguilera, 2016). Para el caso de los piñones de Araucaria angustifolia en Brasil, por ejemplo, y en paralelo a lo que ocurre en el sur de los Andes con las comunidades mapuche-pewenche, el consumo de la semilla de la conífera trasciende lo netamente utilitario y abraza aspectos que modelan la identidad y territorialidad de quienes las utilizan (Reis et al., 2014). En el ámbito nacional, por ejemplo, dos trabajos analizan fases distintas del proceso de producción de productos forestales no madereros. Por su parte, Zúñiga (2013) realiza, desde la perspectiva de la historia oral, una revisión sobre las "rutas de recolección" de los productos más característicos de La Araucanía. El autor visibiliza, 
desde la misma experiencia de los recolectores y recolectoras, las dinámicas socioculturales y económicas asociadas a la recolección. Entre estas dinámicas juegan un papel importante el tiempo, la cantidad de personas que se involucran en el proceso, la importancia de la recolección para la economía familiar, los circuitos dentro del territorio, las formas de almacenar los productos y las distintas formas de comercialización. Por otra parte, Aguilera (2016) no se centra particularmente en los procesos de producción imbricados en los productos, sino en los procesos de valorización que desarrollan productores y clientes. Fundamentalmente, Aguilera (2016) aborda el "boom comercial" en el ámbito gourmet como una apropiación que, entre otras, hace "visibles" a los mapuche como "otros", ya que esa otredad se ha vuelto valor agregado.

El panorama latinoamericano ha desarrollado una economía basada en la explotación de lo étnico-racial de sus pueblos originarios, acompañada de dinámicas extractivitas de recursos naturales, donde el neoliberalismo y sus procesos de comodificación han transformado la forma en que se percibe lo étnico y la naturaleza. Comaroff \& Comaroff $(2011 ; 216$ p) señalan que en una economía de consumo - como la chilena-, donde la identidad social se entremezcla con procesos de comodificación de la otredad, surge una "Etnicidad S.A", la cual la definen como "el contrapunto entre, por un lado, la constitución de la identidad como persona jurídica o ideal y, por otro, la transformación de la cultura en mercancía". Esta transformación es un reto tanto para las comunidades que, entre otras cosas, buscan obtener ganancias económicas de la visibilidad de sus raíces, como también para el mercado global que busca a toda costa la maximización y la venta de estos productos $\mathrm{y}$, a su vez, los alinea frente a lo que es una práctica tradicional.

Se ha sugerido que la metamorfosis de la conciencia neoliberal invade los ámbitos de la vida de los colectivos, los cuales tienden a fortalecer su pertenencia al grupo a medida que ésta pueda traerles beneficios económicos (Comaroff \& Comaroff, 2011). Así, la comunidad establece una lucha por el reconocimiento cultural, pero al mismo tiempo económico (Fraser, 1998), empoderándose y generando procesos de identificación y construcción de un colectivo. Una de las formas de reconocimiento cultural que han adoptado las comunidades, y tal como lo refleja este trabajo sobre el piñón, es el ámbito culinario: las acciones que van desde la distinción entre lo que es alimento y lo que no, pasando por la producción, distribución, preparación y consumo de estos alimentos. Las distintas etapas por las que atraviesa un elemento hasta convertirse en alimento y luego ser consumido, implica distintos actores, trabajos, saberes, poderes y representaciones (Aguilera, 2016). Estas etapas de las que habla Aguilera (2016) están enmarcadas dentro de economías formales. Las economías formales se sustentan en el desarrollo de la teoría de la acción racional, encargada de entender y modelar formalmente el comportamiento social y económico de los agentes. Esta teoría supone que el individuo tiende a maximizar la utilidad-beneficio y a reducir los costos o riesgos.
Respecto al proceso de recolección, éste continúa siendo artesanal y requiere de gran sacrificio físico, pero ha variado su forma con el pasar de los años. El piñón -según su disponibilidad cíclica- es un recurso que se obtiene a través de la recolección, el intercambio o trueque que aún existe, y/o a través de su comercialización por dinero. En algunos casos, las prácticas de recolección están sujetas a la necesidad y a apurar el proceso natural de maduración de semillas, lo que lleva a extracciones poco sustentables, como el corte de ramas y conos. Si bien estas prácticas existen, los recolectores y productores locales son conscientes de los problemas de conservación que aquejan a su árbol y hacen una fuerte crítica a estas prácticas. El turismo parece estar posicionándose como una herramienta para visibilizar y dar a conocer la cultura y paisajes locales, en particular por medio de ofertas gastronómicas con productos de piñón preparados. En esa línea, los usos principales asociados al piñón son de carácter alimenticio, pero conllevan un fuerte componente biocultural y narrativas asociadas al bosque, historia local y tradición.

Existen dos estrategias de comercialización del piñón, una a corto plazo y otra a largo plazo, las que se asocian respectivamente a la venta a granel de piñón y a la venta de productos de piñón elaborados. Distinguimos que existen dos tipos de preparaciones: las preparaciones tradicionales que se heredan por un traspaso generacional, principalmente de la familia; y preparaciones innovadoras dotadas de un sincretismo entre el conocimiento experto formal y el conocimiento experto local. El sistema de comercialización está sujeto a la disponibilidad de piñón, que depende del ciclo de producción de semillas que tiene la araucaria, que también determina los precios. Por ello, se hace referencia a años buenos y años malos, dependiendo de la cantidad de piñón que haya en la temporada. Los productos elaborados de piñón suelen tener precios más estables, lo que reveló lo importante que son las prácticas de conservación del piñón, que no solamente entregan "valor agregado" a la semilla, sino que también permiten abastecerse en años de escasez.

Finalmente, es fundamental recalcar que la elaboración y comercialización de productos de piñón elaborados, tienen asociados procesos en los que participan distintos actores (individuos, familias, empresas, organismos estatales), tanto internos como de fuera de la comuna. Los habitantes de la comuna son conscientes de que el valor del piñón es intrínseco, en tanto que es una semilla característica de su territorio, que los diferencia de otros lugares de Chile. Pero así también es fundamental enfatizar que el trabajo asociado detrás de un producto de piñón no sólo es por la semilla en sí misma, sino por el sacrificio humano que implica la recolección y la elaboración hasta que el piñón llega, en cualquiera de sus formas, a ser consumido. Es necesario un trabajo articulado entre los distintos actores (comunidades, agentes del estado y mercado), que asegure un comercio justo del piñón y medidas de conservación para la regeneración de la araucaria, con el fin de fomentar el bienestar social y ecológico de los bosques de pewen y de las comunidades que recolectan, usan y comercializan esta semilla única del sur de los Andes. 


\section{Agradecimientos}

Agradecemos sinceramente a cada uno de las y los habitantes, tanto colonos como pewenche del territorio de Lonquimay, que compartieron sus espacios, tiempos, conocimientos y prácticas con nosotros. También agradecemos al Centro de Estudios Interculturales e Indígenas (CIIR) CONICYT/FONDAP (15110006), a la Vicerrectoría de Investigación (VRI) de la Pontificia Universidad Católica de Chile a través del proyecto Interdisciplina (7512-023-81), y al Centro para el Impacto Socioeconómico de las Políticas Ambientales (CESIEP) de la Iniciativa Científica Milenio del Ministerio de Economía, Fomento y Turismo (Chile). Agradecemos a Francisca de la Maza y Manuel Gedda, quienes dieron origen a este estudio y facilitaron las gestiones posibles para su desarrollo. También agradecemos a Adrián Fernández, quien preparó la Figura 1, y el aporte de dos revisores anónimos que contribuyeron a mejorar el manuscrito final. Finalmente, reconocemos el acompañamiento y los consejos de Claudia Giacoman.

\section{Referencias}

Aagesen, D.L., 1998. Indigenous resource rights and conservation of the monkey-puzzle tree (Araucaria araucana, araucariaceae): A case study from Southern Chile. Economic Botany, 52: 146. https://doi.org/10.1007/BF02861203

Aguilera-Betti, I., Muñoz, A.A., Stahle, D., Figueroa, G., Duarte, F., González-Reyes, A., Christie, D., Lara, A., González, M.E., Sheppard, P.R., Sauchyn, D., Moreira-Muñoz, A., Toledo-Guerrero, I., Olea, M., Apaz, P. \& Fernández, P., 2017. The First Millennium-Age Araucaria Araucana in Patagonia. Tree-Ring Research, 73 (1): 53-56. https://doi. org/10.3959/1536-1098-73.1.53

Aguilera, I., 2016. De la Cocina al Estado Nación: El Ingrediente Mapuche. Icaria, 320 pp., Santiago.

Barreau, A., 2014. Narrating changing foodways: wild edible plant knowledge and traditional food systems in Mapuche lands of the Andean Temperate Forests, Chile. University of British Columbia, 162 pp., Vancouver.

Barreau, A., Ibarra, J.T., Wyndham, F.S., Rojas, A., \& Kozak, R.A., 2016. How can we teach our children if we cannot access the forest? Generational change in Mapuche knowledge of wild edible plants in Andean temperate ecosystems of Chile. Journal of Ethnobiology, 36 (2): 412 432. https://doi.org/10.2993/0278-0771-36.2.412

Bengoa, J., 2000. Historia del pueblo mapuche siglos XIX y XX. LOM Ediciones, 430 pp., Santiago.

Bengoa, J., \& Valenzuela, E., 1983. Economía mapuche: pobreza y subsistencia en la sociedad mapuche contemporánea. PAS, 121 pp., Santiago.

Bernard, H.R., 2005. Research methods in anthropology: qualitative and quantitative approaches. Altamira Press, 803 pp., Oxford.

Besoain, X.A., Guajardo, J., Larach, A., Riquelme, N., Galvez, E., Tapia, L., Alvarado, L.A., Saratscheff, T., \& Celis-Diez, J.L., 2019. First Report of Diplodia seriata causing gummy canker in Araucaria araucana wild populations in southcentral Chile. Plant Disease, PDIS-01-19-0200-PDN. https://doi.org/10.1094/PDIS-01-19-0200-PDN

CASEN, 2017. Encuesta de Caracterización Socioeconómica Nacional (Casen). Ministerio de Desarrollo Social de Chile, Santiago.
Cockle, K., Ibarra, J.T., Altamirano, T.A., \& Martin, M., 2019. Interspecific networks of cavity-nesting vertebrates reveal a critical role of broadleaf trees in endangered Araucaria mixed forests of South America. Biodiversity and Conservation, In Press. https://doi.org/10.1007/s10531-019-01826-4

Comaroff, J.L., \& Comaroff, J., 2011. Etnicidad S.A. Katz, 251 pp., Barcelona.

Coña, P., 2010. Lonco Pascual Coña ñi tuculpazugun: testimonio de un cacique mapuche. Pehuén, 491 pp., Santiago.

Descola, P., 2013. The ecology of others. Paradigm. Prickly Paradigm Press, 91 pp., Chicago.

Descola, P., \& Gísli, P., 1996. Nature and society: anthropological perspectives. Routledge, 310 pp., London.

Domeyko, I., 2010. La Araucania y sus habitantes. Cámara Chilena de la Construcción, 237 pp., Santiago.

Donoso, C., 2006. Las especies arbóreas de los bosques templados de Chile y Argentina: autoecología. Marisa Cuneo Ediciones, $678 \mathrm{pp}$., Valdivia.

Donoso, S., Peña-Rojas, K., Espinoza, C., Galdámes, E., \& Pacheco, C., 2014. Producción, permanencia y germinación de semillas de Araucaria araucana (Mol.) K. Koch en bosques naturales, aprovechados por comunidades indígenas del sur de Chile. Interciencia, 39 (5): 338-343.

Escobar, A., 1999. After Nature: Steps to an antiessentialist political ecology. Current Anthropology, 40 (1): 1-30. https:// doi.org/10.1086/515799

FIA, 2010. Resultados y lecciones en producción, técnicas de poscosecha y desarrollo de productos a partir del piñón. Fundación para la Innovación Agraria (FIA), 50 pp., Santiago.

Fraser, N., 1998. Iustitia Interrupta Reflexiones críticas desde la posición "postsocialista." Revista de Estudios Sociales, (2): 150-151. https://doi.org/10.7440/res2.1998.29

Friedmann, H., 1980. Household Production and the National Economy: Concepts for the Analysis of Agrarian Formations. The Journal of Peasant Studies, 7 (2): 158-84. doi: $10.1080 / 03066158008438099$.

Gajardo, R., 1993. La vegetación natural de Chile: clasificación y distribución geográfica. Editorial Universitaria, 165 pp., Santiago.

Garibaldi, A., \& Turner, N., 2004. Cultural Keystone Species: Implications for Ecological Conservation and Restoration. Ecology and Society, 9 (3): 1. https://doi.org/10.5751/ES00669-090301

Gedda, M., 2010. Araucanía de Chile. Patrimonio natural y cultural de sus territorios. Editorial Pehuén, 320 pp., Santiago.

González, M.E., Veblen, T.T., \& Sibold, J.S., 2005. Fire history of Araucaria-Nothofagus forests in Villarrica National Park, Chile. Journal of Biogeography, (32): 1187-202. http://doi. wiley.com/10.1111/j.1365-2699.2005.01262.x

Herrmann, T.M., 2005. Knowledge, values, uses and management of the Araucaria araucana forest by the indigenous Mapuche Pewenche people: A basis for collaborative natural resource management in southern Chile. Natural Resources Forum, 29 (2): 120-134. https://doi.org/10.1111/j.14778947.2005.00121.x

Herrmann, T.M., 2006. Indigenous Knowledge and Management of Araucaria Araucana Forest in the Chilean Andes: Implications for Native Forest Conservation. Biodiversity and Conservation, 15 (2): 647-662. https://doi.org/10.1007/ s10531-005-2092-6

Ibarra, J.T., Altamirano, T.A., Gálvez, N., Rojas, I., Laker, J., \& Bonacic, C., 2010. Avifauna de los bosques templados de Araucaria araucana del sur de Chile. Ecología Austral, 20: 33-45.

Ibarra, J.T., Barreau, A., Massardo, F., \& Rozzi, R., 2012. El cóndor andino: una especie biocultural clave del paisaje sudamericano. Boletín Chileno de Ornitología, 18 (1-2): 1-22. 
INE, 2017. Censo poblacional nacional de Chile. Instituto Nacional de Estadísticas, Santiago.

Ingold, T., 2000. The perception of the environment: essays on livelihood, dwelling and skill. Routledge, 480 pp., Londres.

Isla, J., 2001. Pewenche. Estudios sobre territorio y proceso social. Universidad de Chile, 72 pp., Santiago.

Jacques-Coper, A., Cubillos, G. \& Ibarra, J.T., 2019. The Andean Condor as bird, authority, and devil: an empirical assessment of the biocultural keystone species concept in the high Andes of Chile. Ecology and Society, 24 (2): 35. https://doi.org/10.5751/ES-10939-240235

Kerssen, T.M., 2015. Food Sovereignty and the Quinoa Boom: Challenges to Sustainable Re-Peasantisation in the Southern Altiplano of Bolivia. Third World Quarterly, 36 (3): 489507. doi: 10.1080/01436597.2015.1002992.

Kohn, E., 2013. How forests think: toward an anthropology beyond the human. University of California Press, 280 pp., Berkeley.

Maffi, L., 2001. On biocultural diversity: linking language, knowledge, and the environment. Smithsonian Institution Press, 578 pp., Washington DC

Mintz, S.W., 1996. Dulzura y poder: el lugar del azúcar en la historia moderna. Siglo Veintiuno, 299 pp., México DF.

Molina, J., Martín, A., Drake, F., Martín, L., \& Herrera, M., 2015. Fragmentation of Araucaria araucana forests in Chile: quantification and correlation with structural variables. iForest (8): e1-e9. https://doi.org/10.3832/ifor1399-008.

Neira, Z., 1995. La importancia de la semilla de Araucaria araucana para la unidad familiar pehuenche. Revista Desarrollo Agroforestal y Comunidad Campesina, 4 (20): 46-51.

Neri, J.P., 2017. Quinua, comunidad y relaciones capitalistas en el altiplano sur boliviano. Revista de Estudios Bolivianos, 27: $147-167$

Otero, L., 2006. La huella del fuego: historia de los bosques nativos. Poblamiento y cambios en el paisaje del sur de Chile. Editorial Pehuén, 171 pp., Santiago.

Pauchard, A., \& Alaback, P.B., 2004. Influences of evaluation, land use and landscape context on patterns of alien plant invasions along roadsides in protected areas of south -central Chile. Conservation Biology, 18(1): 238-48. https://doi. org/10.1111/j.1523-1739.2004.00300.x.

Perasso, P., 2012. Efectos de la entrega de tierras de CONADI en una comunidad Pehuenche de la comuna de Lonquimay. Universidad de Chile, 121 pp., Santiago.
Plan de Desarrollo Comunal Lonquimay, 2016. PLADECO Lonquimay 2018-2022. Ilustre Municipalidad de Lonquimay, 110 pp., Lonquimay.

Premoli, A., Quiroga, P., \& Gardner, M., 2013. Araucaria araucana. The IUCN Red List of Threatened Species 2013: e.T31355A2805113. http://dx.doi.org/10.2305/IUCN.UK. 2013-1.RLTS.T31355A2805113.en

Reiche, C., 2013. Geografía botánica de Chile. Cámara Chilena de la Construcción, 556 pp., Santiago.

Reis, M.S. dos, Ladio, A., \& Peroni, N., 2014. Landscapes with Araucaria in South America: evidence for a cultural dimension. Ecology and Society, 19 (2): 43. https://doi.org/10.5751/ ES-06163-190243

Sandoval, C., 2002. Investigación cualitativa. ARFO Editores, Colombia.

Schejtman, A., 1980. Economía campesina: lógica interna, articulación y persistencia. Revisa CEPAL, 11: 121-140.

Skewes, J.C., \& Guerra, D.E., 2016. Sobre árboles, volcanes y lagos: algunos giros ontológicos para comprender la geografía mapuche cordillerana del sur de Chile. Intersecciones en Antropología, 17 (1): 63-76.

Speziale, K.L., Lambertucci, S.A., Gleiser, G., Tella, J.L., Hiraldo, F., \& Aizen, M.A., 2018. An overlooked plant-parakeet mutualism counteracts human overharvesting on an endangered tree. Royal Society Open Science, 5 (1): 171456. https://doi.org/10.1098/rsos.171456

Tacón, A., \& Palma, J., 2006. La comercialización de los productos forestales no madereros: una oportunidad para el manejo comunitario y la valorización del bosque nativo. En: Catalán, R., Wilken, P., Kandzior, A., Tecklin, D., \& Burschel, H. (Eds.), Bosques y comunidades del sur de Chile. Editorial Universitaria, 253-266 pp., Santiago.

Wilhelm de Mösbach, E.W., 1992. Botánica indígena de Chile. Editorial Andrés Bello, 120 pp., Santiago.

Zamorano-Elgueta C., Cayuela, L., González-Espinosa, M., Lara, A., \& Parra-Vázquez, M. R., 2012. Impacts of cattle on the South American temperate forests: Challenges for the conservation of the endangered monkey puzzle tree (Araucaria araucana) in Chile. Biological Conservation, 152: 110-8. http://dx.doi.org/10.1016/j.biocon.2012.03.037

Zúñiga, C., 2013. Rutas de recolección en La Araucanía. Printus, $280 \mathrm{pp}$, Temuco. 